\title{
Erratum
}

\section{The Salpeter plasma corrections for solar fusion reactions}

\author{
J. N. Bahcall ${ }^{1}$, L. S. Brown ${ }^{2}$, A. Gruzinov ${ }^{3}$, and R. F. Sawyer ${ }^{4}$ \\ 1 Institute for Advanced Study, Princeton, NJ 08540, USA \\ e-mail: jnb@ias.edu \\ 2 Department of Physics, University of Washington, Seattle, Washington 98195, USA \\ e-mail: brown@.phys. Washington.edu \\ 3 Institute for Advanced Study, Princeton, NJ 08540, USA \\ e-mail: andrei@ias .edu \\ 4 Department of Physics, University of California at Santa Barbara, Santa Barbara, CA 93106, USA \\ e-mail: sawyer@sarek.physics.ucsb.edu
}

A\&A, 383, 291-295 (2002), DOI: 10.1051/0004-6361:20011715

In the paper by Bahcall, J. N., Brown, L. S., Gruzinov, A., \& Sawyer, R. F. 2002, A\&A, 383, 291, the paper by Weiss, A., Fleiskamp, M., \& Tsytovich, N. 2001, A\&A, 371,1123 was wrongly cited as suggesting a modification of the Salpeter formula for screening of nuclear reactions. The main point of the paper by Weiss et al. was actually to show that significant deviations from the Salpeter formula are strongly disfavored by helioseismology measurements. 